\title{
ÉVOLUTIONS DE LA RELATION HOMME/ANIMAL ET ÉTHIQUE PROFESSIONNELLE VÉTÉRINAIRE
}

\author{
HUMAN SOCIETY AND ANIMALS RELATIONSHIP CHANGES AND \\ VETERINARY PROFESSIONAL ETHICS
}

Par André Laurent PARODI ${ }^{1}$

(Communication présentée le 29 avril 2021, manuscrit accepté le 8 juillet 2021)

\begin{abstract}
RÉSUMÉ
S'agissant de l'activité professionnelle vétérinaire, on peut distinguer : l'éthique vétérinaire administrative dont les règles sont définies réglementairement par l'administration de tutelle, l'éthique vétérinaire officielle définie, dans la plupart des pays, par les structures professionnelles ordinales et l'éthique descriptive, enfin, qui répond à des valeurs et des standards moraux laissés au libre arbitre du professionnel. C'est elle qui fera l'objet de cet exposé. Directement commandée par l'ensemble des règles considérées comme bonnes par nos sociétés, elle est, par nature, éminemment temporelle. Elle résulte, à un moment donné de notre histoire, de nos comportements, de nos agissements et de la valeur morale que nous leur accordons. S'agissant de notre pratique professionnelle, ces valeurs ont notablement évolué au cours des dernières décennies avec les modifications profondes du regard que notre société porte désormais sur l'animal, "être sensible" doué de conscience. Qu'il s'agisse de l'animal ressource alimentaire ou force de travail, de l'animal de compagnie ou de laboratoire, désormais, nous ne les considérons plus de la même façon. Un cadre règlementaire régit désormais le "statut de l'animal". Parallèlement, nos compétences techniques et scientifiques ont considérablement progressé, élargissant davantage notre champ opérationnel avec, de ce fait, un libre choix interventionnel accru. L'enseignement et la recherche vétérinaires ont également intégré ces valeurs. Ce sont ces évolutions et leurs effets sur la conduite éthique de notre exercice professionnel qui seront rappelés.
\end{abstract}

Mots-clés : Société, animaux, bien-être animal, médecine vétérinaire, éthique professionnelle.

\begin{abstract}
-ABSTRACT
Three categories of ethics may be recognised in relation with the veterinary professional activity: administrative veterinary ethics which rules are statutory defined by the governing body, official veterinary ethics which is defined in most countries by a professional order and descriptive veterinary ethics which comes up to the personal moral standards of the practitioner. Only the latest will be discussed here. Directly in relation with our society, descriptive veterinary ethics is obviously temporal. On a determined time, it results from our comportments and our moral values. In our professional practice, these values have strongly moved during the last decades according to the changes of our society consideration of animals, "sensitive beings", self-conscious. An animal status is now prescribed for all categories, companion animals, farm animal species and laboratories animals. On the same time veterinary medicine has considerably progressed opening a broader scope of medical competencies. Training and research have also followed these changes. These evolutions and their consequences on the ethical management of veterinary practice are documented.

Keywords: Society, animals, animal welfare, veterinary medicine, professional ethics.
\end{abstract}

1 : Professeur émérite et Directeur honoraire de l'École vétérinaire d'Alfort. Président honoraire de l'Académie vétérinaire de France et de l'Académie nationale de Médecine. Courriel : almparodi@hotmail.com 


\section{INTRODUCTION}

D'une manière générale, "l'éthique est une capacité de raisonnement à la fois logique et morale, qui permet de prendre une décision éclairée en tenant compte d'enjeux moraux. Elle peut être considérée comme la recherche d'une conduite idéale" (Foltzer, 2014). Appliquée à notre exercice professionnel, c'est une valeur qui s'attache à tout acte vétérinaire quel que soit le domaine d'activité concerné. Plusieurs catégories d'éthique professionnelle peuvent être distinguées. S'agissant de l'activité professionnelle vétérinaire, on peut distinguer :

- l'éthique vétérinaire administrative dont les règles sont définies réglementairement par l'administration de tutelle. Toute infraction est passible de sanctions pénales et/ou civiles.

- l'éthique vétérinaire officielle définie, dans la plupart des pays, dont le nôtre, par les structures professionnelles ordinales. Elle repose sur un corpus de normes et de valeurs imposées à la profession. Les manquements peuvent conduire à des sanctions disciplinaires.

- l'éthique descriptive enfin, qui répond à des valeurs et des standards moraux laissés au libre arbitre du professionnel. Elle n'est pas justiciable de sanctions. C'est elle qui fera l'objet de cet exposé.

Directement commandée par l'ensemble des règles considérées comme bonnes par nos sociétés, elle est, par nature, éminemment temporelle. Elle résulte, à un moment donné de notre histoire, de nos comportements, de nos agissements et de la valeur morale que nous leur accordons. S'agissant de notre pratique professionnelle, ces valeurs ont notablement évolué au cours des dernières décennies avec les modifications profondes du regard que notre société porte désormais sur l'animal, "être sensible" doué de conscience. Qu'il s'agisse de l'animal ressource alimentaire ou force de travail, de l'animal de compagnie ou de l'animal de laboratoire, nous ne les considérons plus aujourd'hui de la même façon qu'il y a encore quelques décennies. En outre, désormais, un cadre règlementaire régit le "statut de l'animal". Parallèlement à l'évolution de nos mentalités, les compétences techniques et scientifiques de notre profession ont considérablement progressé, élargissant davantage notre champ opérationnel avec, de ce fait, un libre choix interventionnel accru. L'enseignement et la recherche vétérinaires ont également intégré ces valeurs. Ce sont ces évolutions et leurs effets sur la conduite éthique de notre exercice professionnel qui seront analysés.

\section{UNE ÉVOLUTION DU REGARD QUE NOTRE SOCIETÉ PORTE SUR L'ANIMAL}

Certes le sentiment de compassion qu'inspirent les mauvais traitements infligés aux animaux n'est pas entièrement nouveau. Dès 1824, en Angleterre, est créée la Society for Prevention of Cruelty to Animals, ancêtre de notre SPA. Peu de temps après, en 1845, en France, le Docteur Etienne Pariset, créait la Société pour la Prévention de la Cruauté envers les Animaux. Chacun connait la Loi du 2 juillet 1850 inspirée par le général Jacques Delmas de Grammont, "sur les mauvais traitements envers les animaux domestiques", destinée à faire cesser les brutalités infligées aux chevaux par certains cochers, sur la voie publique. Mais il faut attendre 1976 avec l'Article L 214-1 du Code Rural et la Loi 76-629 du 10 juillet 1976 pour que le législateur impose que "Tout animal étant un être sensible doit être placé par son propriétaire dans des conditions compatibles avec les impératifs biologiques de son espèce". et précise qu'"Il est interdit d'exercer des mauvais traitements envers les animaux domestiques". A la suite d'un long débat parlementaire, un nouvel article a été introduit dans la code civil (article 515-14) qui stipule que "les animaux sont des êtres vivants doués de sensibilité" tout en précisant toutefois que "Sous réserve des lois qui les protègent, les animaux sont soumis au régime des biens".

La notion de "bien-être animal" (BEA) est désormais unanimement acceptée. Au delà d'un bon état de santé, on reconnaît à l'animal la faculté de jouir d'un environnement adapté à son espèce. Largement médiatisée, elle répond selon un sondage IFOP, à une attente des deux tiers de la population de notre pays (Nippert, 2020). Un groupe d'étude parlementaire dédié à la condition animale a été créé à l'Assemblée nationale. Présidé par notre confrère le Docteur Loïc Dombreval, il est à l'origine d'une proposition de loi visant à renforcer la lutte contre la maltraitance animale, adoptée par l'Assemblée nationale le 29 Janvier 2021. Au Sénat, a été également créé un groupe d'étude "Élevage" dont la vice-présidence a été confiée à un autre de nos confrères, le Dr Arnaud Bazin, chargé plus spécialement de la thématique "Animal et Société". "Placer le bien-être animal au coeur d'une activité durable" est devenu l'objectif de la nouvelle stratégie nationale que le Ministère de l'Agriculture, de l'Agroalimentaire et de la Forêt a fixée, dès 2016, de manière à répondre à l'attente sociétale. Il en est résulté une liste de préconisations mises en place pour 5 ans. Le Conseil économique, social et environnemental a d'ailleurs émis un avis (27 novembre 2019) fixant ses recommandations en la matière. L'Organisation mondiale de la santé animale (OIE) a donné également une définition du bien-être animal. Depuis le 1er février 2020, l'Agence nationale de sécurité sanitaire de l'alimentation, du travail et de l'environnement (ANSES) est membre du Centre national de référence et coordonnateur du Centre européen pour le bien-être des volailles, lapins et autres petits animaux d'élevage, en collaboration avec les organismes partenaires en Espagne, Italie et Danemark (EURCAW-SA). Dans un avis du 26 avril 2018, l'Agence proposait une définition du BEA et définissait le socle de ses travaux de recherche et d'expertise en s'appuyant sur une mise en perspective philosophique, sociétale et juridique du concept. S'agissant du bien-être des animaux d'élevage, une nouvelle approche figure dans cet avis ; baptisée "One welfare" elle implique que le bien-être de ces animaux est étroitement lié à celui de ceux et celles qui en ont la charge. Bien entendu le vétérinaire devrait tenir une place centrale dans cette démarche en raison de sa position. Comme nous le verrons, cette faculté l'expose naturellement à des contraintes d'ordre éthique.

Allant plus avant et à la demande de l'Autorité européenne de sécurité des aliments (EFSA), une expertise sur la conscience animale a été menée par l'Institut national de la recherche agronomique et environnementale (INRAe) (Le Neindre et al. 2018). Il ressort de cette expertise que les animaux, les mammifères au moins et certaines espèces d'oiseaux, ont une 
capacité à éprouver des émotions, à gérer des situations complexes et à évaluer des savoirs propres. Ils peuvent aussi planifier des actions par rapport à leur expérience et gérer des relations sociales complexes entre eux et avec les humains. La mise en convergence de l'ensemble de ces connaissances permet d'argumenter en faveur de l'existence de formes de conscience chez certains animaux. Le terme de "sentience" a été créé pour désigner cette forme de conscience. Si le statut d'être conscient ne se discute plus dans notre société, s'agissant des espèces animales dites de compagnie pour lesquelles les termes de "clients" et de "propriétaire" ("pet owner") ont désormais été remplacées par ceux de "patient" et de "pet parents", la question demeure plus controversée pour les animaux d'élevage où le débat reste ouvert en vue d'améliorer leur bien-être physique et mental et limiter les douleurs sans, pour autant, trop s'affranchir des contraintes économiques. Bien entendu, ce même débat existe à un degré passionnel plus élevé, avec les animaux dits de laboratoire. Enfin il n'est pas inutile de rappeler que, face à ces nouvelles situations professionnelles, le Conseil national de l'ordre des vétérinaires (CNOV) s'est doté depuis 2018 d'un Comité d'éthique animal, environnement, santé. Fidèle aux définitions que nous donnions au tout début, l'Ordre entend avec l'appui de ce Comité, délivrer des avis ou des recommandations. Cependant, il restera toujours au praticien à se déterminer en fonction de ses propres valeurs éthiques.

\section{DES PROGRÈS SPECTACULAIRES EN MÉDECINE VÉTÉRINAIRE}

Au cours des trois dernières décennies, la médecine vétérinaire opérationnelle a fait des progrès considérables, inspirés des progrès de la médecine de l'Homme. Les soins dispensés aux animaux, en particulier d'espèces dites de compagnie, connaissent un niveau interventionnel souvent comparable à celui de la médecine. Des chapitres nouveaux ont été ouverts grâce aux progrès de l'imagerie médicale et de l'anesthésiologie notamment. L'oncologie vétérinaire, l'orthopédie avancée, la neurochirurgie, l'immunologie, voire les greffes d'organes sont désormais entrés ou vont le faire, dans la pratique. Ces soins avancés ont un coût. L'Animal Pet Product Association indique qu'aux États-Unis, en 2019, les dépenses engagées au bénéfice des animaux de compagnie ont atteint près de cent milliards de dollars (95,7 milliards de \$) dont 29,3 Mds pour les soins et produits vétérinaires. Il est intéressant de noter que la possession d'un animal de compagnie est corrélée aux revenus : 60\% des foyers dont le revenu est supérieur à $80000 \$$ possèdent un animal, contre seulement $36 \%$ des foyers gagnant moins de 20000 \$. Il faut noter aussi que, selon un rapport du National Bureau of Economic Research, cité par AVMA News, les dépenses liées aux soins vétérinaires, toujours aux États-Unis, progressent au même rythme que les dépenses de santé humaine. Entre 1996 et 2012 le chiffre de la progression a été de $50 \%$ pour la santé humaine et de $60 \%$ pour la santé des animaux. Le même rapport indique que les individus gagnant plus de $70000 \$$ par an dépensent deux fois plus pour leurs animaux de compagnie que ceux dont les revenus sont de $20000 \$$.

\section{UNE ÉTHIQUE PROFESSIONNELLE VÉTÉRI- NAIRE EN ÉVOLUTION}

Ces glissements sociétaux et médicaux vétérinaires ont forcément modulé notre éthique professionnelle. Très naturellement, ils l'ont fait avec des degrés d'adaptation quelque peu différents selon le cadre d'exercice professionnel. Nous nous attacherons aux activités vétérinaires en médecine des animaux de compagnie, à celle des animaux d'élevage et finalement à l'expérimentation animale.

\section{Médecine vétérinaire des animaux de compagnie}

L'éthique professionnelle que nous avons qualifiée de descriptive, se caractérise par le fait qu'elle implique trois partenaires : le praticien, son patient animal bien entendu et aussi le propriétaire de cet animal. On a pu dire qu'elle partage cette particularité avec la pédiatrie.

Nous tenterons de l'analyser dans trois circonstances : les choix thérapeutiques, les interventions nécessitant un investissement financier élevé et l'euthanasie.

- Des choix thérapeutiques : lorsque j'ai commencé mes activités dans la chaire d'Anatomie pathologique à l'École d'Alfort, l'euthanasie était la destinée quasi automatique des chiens et des chats atteints d'un cancer évolué. Désormais, l'oncologie vétérinaire est une discipline clinique à part entière et connaît de succès spectaculaires. Il existe des Centres d'oncologie animale. Néanmoins, comme l'écrivait récemment notre confrère le Dr Jean-Pierre Samaille, " faut-il traiter un chien souffrant d'un hémangiosarcome splénique métastatique par la Doxorubicine à forte dose, sachant qu'on va le rendre malade et ce pour gagner au mieux 50 jours de survie, dans des conditions épouvantables. Doit on amputer un chien souffrant d'un ostéosarcome et lui infliger de surcroît une chimiothérapie sachant qu'il va certainement entrer dans une phase généralisée de son cancer? " (Samaille, 2019). Sans compter, en outre, les contraintes strictes à observer au cours des cures de chimiothérapie. Dans ce cas, l'éthique professionnelle passe naturellement par la demande de son consentement éclairé au propriétaire ; celui-ci devra être soigneusement et très honnêtement informé de la balance entre, d'une part, le coût financier du traitement ainsi que son caractère contraignant et pénible pour l'animal et, d'autre part, les résultats escomptés. Une publication canadienne récente commente les différents modes de communication entre propriétaires et vétérinaires à travers l'analyse des échanges au sein de groupes de discussion de propriétaires et de vétérinaires (Janken et al. 2021). Comme on pouvait le prévoir, elle révèle qu'une prise de décision dite "partagée", c'est à dire qui résulte de la fourniture des renseignements demandés par le propriétaire et qui est prise de concert, est largement préférée aux prises de décision dites "paternaliste", lorsque le praticien s'est borné à une communication unidirectionnelle, ou même "éclairée" lorsque le vétérinaire fournit les renseignements demandés par le propriétaire, lequel prend sa décision de manière autonome, sans recevoir de directives ou de conseils de la part du praticien. Un groupe de travail, créé au sein de l'European College of Veterinary Anaesthesia and Analgesia (ECVAA) par des confrères universitaires autrichiens, s'est penché sur ce qui risquerait de devenir de 
l'acharnement thérapeutique (Grimm et al. 2018). Les conclusions sont qu'en toutes circonstances, des principes moraux doivent sous-tendre la décision, celle-ci pouvant conduire le praticien à proposer l'euthanasie ; tout acte doit être pesé et expliqué sur la base de considérations éthiques et morales et non pas du profit voire de la vanité. Ce groupe de travail a convenu de développer un "Veterinary Ethic Tool" (VET), fournissant une aide à la prise de décision des praticiens. La règle unanimement admise dans notre profession est que la réponse réside dans l'intérêt de l'animal. Un autre groupe de confrères autrichiens faisait les mêmes réserves à propos de défis éthiques soulevés par de nouvelles avancées thérapeutiques et chirurgicales telles que prothèses de hanche, remplacement de valvules cardiaques, dialyses et bien entendu cancérologie (Springer et al. 2019). Beaucoup parmi eux estimaient que leur "client" est avant tout l'animal et non son propriétaire. Ils regrettaient l'attitude de certains propriétaires jusqu'au-boutistes et plus encore peut être pour notre propos, celle parfois de confrères, jeunes généralement, imprégnés de la formation reçue au cours de leurs récentes études, qui se comportent davantage comme des techniciens de talent que comme des praticiens responsables. Plus délicat encore car ne compromettant pas la santé immédiate de l'animal est celui des interventions dites de convenance. Il y a quelques années, m'exprimant devant les étudiants de notre École sur ce sujet, j'évoquais une information de la presse généraliste, faisant état d'un projet de décret émanant d'une sénatrice américaine, visant à limiter les interventions de chirurgie esthétique pratiquées généralement chez des chiens en vue de corriger un état d'obésité et dont le coût s'élevait à plusieurs millions de dollars (Parodi, 2017). Je trouvais ce projet effectivement judicieux. Jusqu'à ce que poursuivant ma lecture, je découvrais que l'argument premier n'était pas le caractère excessif des dépenses, mais le fait qu'il ne paraissait pas moral (éthique) aux yeux de la sénatrice, d'infliger des souffrances à ces animaux au seul motif de satisfaire une certaine vanité d'ordre esthétique de leurs propriétaires ! Ce même débat conduit à soulever la question de ce que l'on appelle les soins onéreux.

\section{Les soins onéreux ou la Médecine vétérinaire " à deux vitesses "}

Ici encore, je vais faire référence à une information relevée dans la grande presse professionnelle (Parodi, 2017). Elle rapportait l'intervention réparatrice survenue sur un chien King-Charles appartenant à un couple d'Américains. Pratiquée dans une clinique versaillaise, de manière à éviter au chien une longue quarantaine japonaise car l'éminent chirurgien cardio-vasculaire, invité tout exprès, était japonais, l'intervention s'était soldée par une facture de 40000 \$! Sans vouloir refuser à ce couple la satisfaction d'avoir soulagé leur "enfant", elle évoque la situation des médecins britanniques qui doivent évaluer les conditions auxquelles doit satisfaire un patient pour pouvoir, ou non, bénéficier d'un "traitement onéreux" en fonction de son état ou de son âge. Le risque d'une médecine vétérinaire "à deux vitesses" s'est introduit dans notre pratique. De plus en plus, notamment à la faveur du développement de l'assurance santé, ces dilemmes d'ordre éthique deviendront plus fréquents.
Que penser encore du clonage "commercial" des chiens et des chats de compagnie? Une société Nord-Américaine produit à la demande et en conformité avec la législation américaine, des animaux génétiquement identiques à un animal disparu. Bien entendu, cela a un coût, celui du prélèvement tissulaire et de sa conservation en vue du clonage futur qui s'élève à 1600 \$ et celui du clonage lui-même qui revient à environ 50000 \$ pour un chien et $25000 \$$ pour un chat! (Brown, 2017).

\section{L'euthanasie}

Dans notre pays, un vétérinaire se trouve confronté, en moyenne, à 60 fins de vie animale par an : $15 \%$ par mort naturelle et $85 \%$ par euthanasie (Simeon, 2019). La France est le seul pays européen à bénéficier de services de crémation ou d'inhumation ouverts aux animaux familiers décédés dans une clinique vétérinaire. Il existe même des centres de ressources qui assurent un véritable service de pompes funèbres pour nos animaux de compagnie. Alors que l'euthanasie a été jusque vers les années 80 un acte banal, servant même à l'apprentissage des injections intraveineuses dans nos Écoles, c'est devenu un acte empreint de solennité, auquel les propriétaires sont invités à assister, souvent dans un local dédié de la clinique vétérinaire. Cette gravité implique une attitude éthique de la part du vétérinaire. Au delà des gestes techniques, le praticien, outre l'organisation de la prise en charge des corps, se doit d'expliquer aux propriétaires, en la justifiant, cette décision ultime. Il se doit de ne pas juger les choix de "la famille", pour autant qu'ils ne soient pas en contradiction avec les règles déontologiques ou ses valeurs éthiques. À ce sujet, une publication récente fait état des résultats d'une enquête menée aux États-Unis auprès des étudiants d'établissements d'enseignement vétérinaire, de laquelle il ressort que l'acte d'euthanasie ne bénéficie pas d'un enseignement adapté. Les conclusions sont qu'une amélioration et une standardisation des pratiques sont hautement souhaitées (Cooney, 2021).

L'identification d'actes de maltraitance au cours d'une consultation, soulève encore des questions d'éthique. Sans en arriver aux cas de mise en danger grave pour l'animal, tels qu'animaux dénutris, privés d'eau... qui, selon les termes du code rural, contraignent le praticien à "informer sans délai l'autorité administrative ", certains manquements à la couverture des besoins élémentaires des animaux, des lésions révélatrices d'actes de cruauté, voire de zoophilie, soulèvent la question de la violation du secret professionnel imposé par les règles déontologiques professionnelles. Ce qui constitue un délit. Dans de tels cas, la solution pourra passer par une information adressée à l'Ordre (Corlouer, 2017). Ces mêmes questions d'ordre éthique se posent, bien entendu et plus souvent peut être encore, en médecine des animaux d'élevage.

\section{Médecine vétérinaire des Animaux d'élevage}

Bien que, comme nous l'avons rappelé, les espèces animales dites d'élevage, mammifères ou oiseaux, bénéficient également du statut "d'êtres sensibles", les contraintes économiques qui pèsent sur leur production, compliquent fortement l'application de ce statut. Il n'en est pas moins certain que des progrès 
réels sont en cours, notamment dans l'élevage avicole. Dans notre pays, le Conseil de l'Ordre des vétérinaires rappelle que les Docteurs vétérinaires, professionnels de la santé animale, contribuent à construire les bases scientifiques nécessaires à la recherche de solutions pérennes et pragmatiques en vue d'améliorer la prise en compte du bien-être animal. Le respect dû aux animaux est un devoir déontologique. Certes, émanant du Conseil de l'Ordre, ces recommandations s'inscrivent davantage dans ce que nous avons défini comme étant l'éthique vétérinaire officielle. Néanmoins, par sa présence au sein des élevages, par les relations très généralement de confiance qu'il établit avec les éleveurs, le vétérinaire est à même de rechercher avec eux des orientations, voire des solutions visant à améliorer la condition des animaux. Les modalités de l'abattage des animaux de boucherie fait l'objet de débats souvent tendus, alimentés par le militantisme de certaines associations. Sont régulièrement dénoncés, la brutalité des pratiques industrielles ainsi que les méthodes cultuelles de mise à mort sans étourdissement. Un Comité national d'éthique des abattoirs mis en place à l'initiative d'une commission d'enquête sur les conditions d'abattage créée à l'Assemblée nationale, a pour objectif de "Réaliser une analyse des attentes sociétales, donner un avis sur la politique publique, débattre de l'évolution de la législation et de la réglementation relatives à l'amélioration de la protection animale en abattoir et jouer un rôle dans le suivi de leur mise en œuvre". Il est actuellement présidé par le Président de l'Académie vétérinaire de France. L'Ordre des vétérinaires, soutenu avec persévérance par l'association "Euvre d'assistance aux bêtes d'abattoir" (OABA) a émis un avis en 2015, stipulant que "tout animal abattu doit être privé de conscience de manière efficace préalablement à la saignée et jusqu'à la fin de celle-ci". S'agissant de l'abattage dit rituel soumis aux dispositions dérogatoires prévues dans le Droit européen et qui sont accordées aujourd'hui dans le Droit français au nom du respect de la liberté de religion, des confrères ont obtenu, au titre de leur éthique professionnelle, que dans certains abattoirs ayant reçu dérogation, le délai entre l'égorgement et l'étourdissement post-égorgement soit raccourci. Un système lumineux permet de contrôler ce délai. Pour en terminer avec la pratique vétérinaire dans le milieu de l'élevage, bien qu'il s'agisse d'une situation hybride, il convient d'évoquer la médecine équine. En terme d'éthique, une question particulière se pose au praticien lorsque le propriétaire d'un cheval ne peux plus ou ne veut plus continuer à l'entretenir. Deux solutions s'offrent à lui : l'abattage en vue de la boucherie chevaline ou l'euthanasie. Cette situation est souvent généra- trice d'un conflit moral pour le praticien, partagé entre l'euthanasie de convenance d'un animal en bonne santé et les réserves émises par le propriétaire, rebuté par l'idée d'envoyer son animal à l'abattoir. Il est unanimement reconnu que son choix éthique orientera le praticien vers l'abattage (Rémy, 2016).

\section{L'expérimentation animale}

Les progrès scientifiques et médicaux ont nécessité et nécessitent toujours le recours à l'animal de laboratoire. Régulièrement révisée, une réglementation encadre très fermement le recours à l'animal de laboratoire (Parodi, 2009). Cette règlementation, régie à la fois par les instances nationales, en l'occurrence le Ministère chargé de l'Agriculture, et la Commission européenne, impose la prise en considération de règles éthiques auxquelles sont soumis les expérimentateurs. Toute expérimentation doit recevoir, préalablement à sa mise en ouvre, l'avis d'un Comité d'éthique dont fait obligatoirement partie un vétérinaire. Bien qu'il s'agisse de ce fait de ce que nous avons considéré comme une éthique administrative, il est évident que ce vétérinaire interviendra, au moment de l'examen des protocoles expérimentaux par le Comité d'éthique, dans le sens de l'épargne de la souffrance animale. Une attention particulière sera portée à la définition des "points limites" fixant le degré de contrainte douloureuse imposée lorsque le protocole l'exige, au delà duquel, l'expérimentation doit cesser.

\section{CONCLUSION}

Le regard que nos sociétés portent sur l'animal a considérablement évolué au cours des dernières décennies. Désormais, l'animal, quelle que soit son espèce, est considéré comme un être sensible et à ce titre, nous nous devons de lui assurer un état de bien-être. Parallèlement, l'espace technique et scientifique de la médecine vétérinaire s'est considérablement élargi, ouvrant au praticien des choix d'intervention multiples. Paradoxalement en apparence, ces avancées ne sont pas toujours génératrices d'un confort absolu pour le praticien. Elles créent des situations parfois complexes dont la solution est principalement guidée par des valeurs d'ordre éthique. Sources de tensions émotionnelles pour le praticien, elles justifient une formation appropriée que la plupart de nos établissements d'enseignement vétérinaire ont d'ores et déjà développée. Enfin, la concertation au sein des cliniques vétérinaires est un moyen facilitant la démarche permettant de les résoudre.

\section{BIBLIOGRAPHIE}

- Brown J. Pet cloning gives clients an opportunity to recapture the past, though ethical concerns linger. Veterinary Practice News. 2017. Disponible à : https://www.veterinarypracticenew .com (Consulté le 18.2.2021).

- Cooney K. Dickinson G E. Hoffmann H. Euthanasia Education in Veterinary Schools in the United States. J Vet
Med Educ. 2021. Disponible à : e20200050, DOI: $10.3138 /$ jvme-20200050. (Consulté le 12.3.2021).

- Corlouer N. Maltraitance animale et rôle du vétérinaire : entre Éthique et respect de la loi. La Semaine Vétérinaire. $2017 ; 52-57$.

- Foltzer M. Contribution à l'étude de l'enseignement de l'éthique dans les établissements d'enseignement vétérinaire européens. Thèse de médecine vétérinaire, Vet Agro Sup. 2014.

- Grimm H. Bergadano A. Musk G C. Otto K. Taylor P M. Duncan J C. Drawing the line in clinical treatment of companion animals: recommendations from an ethics working party. Veterinary Record. 2018. Disponible à: 
http://dx.doi.org/10.1136/vr.104559 (consulté le 25. 3 2021).

- Janken N, Jason B, Coe J B, Bernardo T M. Dewey C E. Stone E A. Pet owner's and veterinarians' perception of information exchange and clinical decision-making in companion animal practice, PLOS One. 2021; 16, e0245632.

- Le Neindre P, Dunier M, Larrère R, Prunet $\mathrm{P}$. La conscience des animaux. Versailles : QuAE éditeur ; 2018.
- Nippert A. Deux tiers des Français en faveur du bien-être animal. Le Monde. 20 Août 2020 ; 11.

- Parodi A L. Éthique en expérimentation animale. Bull. Acad. Nat. Méd., 2009 ; 193 : 1737-1746, séance du 10 novembre 2009.

- Parodi A L. Exercice vétérinaire et éthique professionnelle. La Semaine Vétérinaire. 2017 ; 63- 64.

- Rémy D. Quel devenir pour un cheval que son propriétaire ne peut pas continuer à entretenir ? analyse éthique. Le Point Vétérinaire. 2016 ; 70-73.

- Samaille J P. Jusqu'où allons-nous trop loin? L'Essentiel. 2019 ; 536 : 5.

- Simeon C. Enrichir la fin de vie. Parce que la mort est aussi notre métier. L'Essentiel. 2019; 45-46.

- Springer S. Sandøe P. Bøker Lund T. Grimm H. Patients' interests first, but ... Austrian Veterinarians' attitudes to moral challenges. Animals (Basel). 2019; 9: 241. 